\title{
COMMUNICATION
}

\section{Dysplasies (cémento-) osseuses florides : présentation clinico-radiologique d'une série de cas et diagnostic différentiel avec les lésions inflammatoires pẻri-radiculaires d'origine endodontique LIPOE}

\author{
Daviet-Noual $\mathbf{V}^{1}$, Moreau $\mathbf{N}^{1,2}$, Gossiome $\mathbf{C}^{1}$, Ejeil AL' ${ }^{1}$, Salmon $\mathbf{B}^{1,3}$ \\ 1- AP-HP - Hôpitaux Universitaires Paris Nord Val de Seine, Services d'Odontologie Bretonneau, Paris, France \\ 2- Labo NM
}

3- EA 2496, Université Paris Descartes Sorbonne Paris Cité, Faculté de Chirurgie Dentaire, Montrouge, France

\section{Introduction}

Les dysplasies (cémento-) osseuses florides DOF sont des lésions dysplasiques fibro-osseuses classées parmi les tumeurs odontogènes bénignes (OMS 2005) avec les dysplasies osseuses focales, péri apicale et le cémentome gigantiforme familial.

Elles surviennent typiquement chez une femme d'âge moyen et d'origine africaine.

Cette affection est généralement asymptomatique et sa découverte le plus souvent fortuite lors d'un examen radiologique dentaire de routine. Un CBCT peut être utile pour orienter le diagnostic. II s'agit de lésions d'évolution lente classiquement multiples bilatérales et symétriques atteignant les régions dentaires péri-apicales maxillaires et mandibulaires avec une prédominance mandibulaire. Trois stades radiologiques successifs sont classiquement décrits : radioclaires puis mixtes et enfin homogènes radioopaques avec un halo radioclair périphérique. Au stade initial les lésions radiologiques peuvent être confondues avec celles des lésions inflammatoires péri-radiculaires d'origine endodontique (LIPOE), ce qui peut conduire à un traitement radiculaire inapproprié. Le test de vitalité pulpaire est l'examen essentiel et discriminatif dans le diagnostic différentiel.

\section{Observation}

Nous présentons une série de cas de DOF typiques décrits à différents stades d'évolution radiologique : phase initiale, mixte et tardive documentés par panoramique dentaire et CBCT. L'attitude thérapeutique a été axée sur une surveillance clinique et radiologique simple.

\section{Discussion}

La DOF une affection d'évolution lente, le diagnostic est clinique et surtout radiologique. Au stade initial le diagnostic différentiel avec les LIPOE est nécessaire pour éviter un traitement endodontique non justifié susceptible de surinfecter les lésions. Aux stades plus avancés le diagnostic différentiel avec d'autres lésions ossifiantes se discute notamment avec le fibrome ossifiant, la maladie de Paget et l'ostéomyélite chronique diffuse. Le fibrome ossifiant se distingue de la DOF par son aspect plus limité et encapsulé ainsi qu'un effet de masse sur les racines voire leur

This is an Open Access article distributed under the terms of the Creative Commons Attribution License 4.0, which permits unrestricted use, distribution, and reproduction in any medium, provided the original work is properly cited. 
résorption. Pour la maladie de Paget l'atteinte osseuse est diffuse, sans images radioclaires au sein des opacités et avec une hausse de phosphatases alcalines. L'ostéomyélite chronique diffuse associe douleur, tuméfaction unilatérale et atteinte osseuse au-delà des secteurs dentaires. Certaines patientes présentent une DOF associée à un kyste osseux solitaire.

L'imagerie conventionnelle apporte généralement suffisamment d'indication radiographique pour poser le diagnostic et l'examen CBCT est non justifié lorsque non contributif au diagnostic ou à la prise en charge. La biopsie pour examen histologique des lésions de DOF est inutile pour le diagnostic et potentiellement iatrogène.

En l'absence de symptomatologie clinique aucun traitement n'est nécessaire. Les patients doivent être informés du caractère quiescent des lésions, de la nécessité d'une abstention thérapeutique et d'une surveillance pour réévaluer les lésions et prévenir les surinfections. Pour les cas symptomatiques les solutions thérapeutiques reposent sur une antibiothérapie dont l'efficacité est limitée en raison du caractère avasculaire des lésions et les chirurgies de résection et de remodelage.

\section{Conclusion}

La DOF est affection bénigne d'évolution lente et quiescente dont la prise ne charge est axée sur une surveillance clinique et radiologique. II est essentiel d'en faire le diagnostic pour éviter les traitements radiculaires inappropriés.

\section{Références}

1/ M.Heuberger B et al. Rev Mens Suisse Odontostomatol 2010, Vol. 120 1007-1011.

2/ Gündüz K et al. Braz Dent J 2009, Vol. 20 347-50.

3/ Dghoughi S et al. Rev Odont Stomat 2010, Vol. 39 211-221. 